Digital scarcity

Volume 10 Issue 2

GLOSSARY

\title{
Digital scarcity
}

Jaya Klara Brekke Durham University j.k.brekke@durham.ac.uk

Aron Fischer Colony aron@colony.io

DOI: https://doi.org/10.14763/2021.2.1548

Published: 19 April 2021

Received: 25 November 2021 Accepted: 8 December 2020

Competing Interests: The author has declared that no competing interests exist that have influenced the text.

Licence: This is an open-access article distributed under the terms of the Creative Commons Attribution 3.0 License (Germany) which permits unrestricted use, distribution, and reproduction in any medium, provided the original work is properly cited. https://creativecommons.org/licenses/by/3.0/de/deed.en Copyright remains with the author(s).

Citation: Brekke, J. K. \& Fischer, A. (2021). Digital scarcity. Internet Policy Review, 10(2). https://doi.org/10.14763/2021.2.1548

Keywords: Blockchain

Abstract: Digital Scarcity is a credibly maintained limitation, imposed through software, of digital information, goods or services that may be accessed and used entirely digitally. 
This article belongs to the Glossary of decentralised technosocial systems, a special section of Internet Policy Review.

\section{Definition}

Digital Scarcity is a credibly maintained limitation, imposed through software, of digital information, goods or services that may be accessed and used entirely digitally.

\section{The history of digital scarcity}

Some of the earliest uses of the term digital scarcity stem from the early 2000s and describe the scarcity of access to IT resources and the underlying physical resources that computers and networks rely on-i.e. "the scarcity of the digital" (Weinberger, 2003; Hammersley, 2003; Chaudhry \& Shipp, 2005). In one prominent example, legal restrictions on access to radio frequencies stymied the growth of communications networks at the beginning of this century-and activists and network operators bemoaned the resulting digital scarcity (Weinberger, 2003; Hammersley, 2003). The explosive growth of mobile telephony and the widespread demand for digital services on mobile devices led to public auctions of radio spectrum usage rights in order to alleviate this particular form of legally imposed scarcity (Wikipedia contributors, 2021). Digital scarcity, as referring to the availability of IT resources, has also described issues of accessibility due to forms of marginalisation (the digital divide), including lack of access because of disabilities. In this specific context, the term digital scarcity is used to describe "the dearth of accessible technological and other resources, lack of political will to address the problem, and general ignorance about digital access" (Chaudhry \& Shipp, 2005, sect. 6, para. 10).

As internet access has become more widespread, and as an increasing amount of content is consumed digitally (text, news, music), the usage of the phrase has shifted. Digital scarcity now refers to the imposition of limits and conditions on availability and access to digital content. Digital information can be easily copied and is by nature not scarce or rivalrous; it can be shared at next to no cost, with no reduction in availability or quality: 'Digital reproduction frustrates notions of originality' (O'Dwyer, 2020, p. 874). As assets that were previously scarce or rivalrous (e.g., number of copies of a book or record) became increasingly digital, this led to no end of difficulties in the realm of copyright enforcement (Perzanowski \& 
Schultz, 2016). In this context, digital scarcity describes limitations set on the access to data, in order to protect business models that depend on scarcity, as well as in the development of new forms of digital markets and economies.

Copyright-based industries for example, seek to impose digital scarcity to prevent copying of data. In the case of music, the value to the customer is clear, but scarcity needs to be maintained in order to protect industry profits. In the words of Warner Music Group chairman Edgar Bronfman: "Music is ubiquitous to a degree that also I think is probably not helpful for the industry" (Resnikoff, 2007). On the other hand, manipulating (perceptions of) scarcity is used a priori as a marketing tool in order to create demand for digital goods and services (Fortin, 2007). This (pre-bitcoin) notion of scarcity is perhaps best summed up in the following: "One key to the success of digital goods business models is to maintain the scarcity of the digital goods. Since digital goods are digital, they cost nothing to copy. Free copies of digital goods would reduce demand for paying for the same item. In a closed system, it is easier to maintain scarcity. The company controls the supply of all digital goods completely." (Lightspeed Venture Capital Partners, 2008).

The rise of the internet, and the ease with which data could be copied, led to movements of digital activists seeking to open the access to information entirely ('information wants to be free'). These movements often clashed with intellectual property and copyright-based industries (Dahlstrom et al., 2006; Swartz, 2016), and these clashes in turn informed much of the development of peer-to-peer systems that would enable circumvention of the copyright industry, and free access to information (Oram, 2001; Andersson, 2011). Arguments were put forward that nothing digital is genuinely scarce and that any imposed scarcity is not just artificial, but also objectionable. "In digital goods, scarcity doesn't exist" and "the economics of scarcity doesn't apply digitally" (Masnik, 2006a). Virtual goods are only scarce by design and, as such are scarce by choice, and "that's a recipe for trouble" (Masnik, 2006b; see also Knowles, Castronova, and Ross, 2015, p. 242).

If we look beyond mere data, there are digital resources that are inherently limited, such as bandwidth or short domain names. Short domain names for instance (using the English alphabet) are scarce due to the limitations of the alphabet, and may sell for millions of dollars (WP02); but scarcity of top-level-domains (such as .com, .org, .luxe, or .io) is artificially created and maintained by the Internet Assigned Numbers Authority (IANA) and the Internet Corporation for Assigned Names and Numbers (ICANN). Sometimes the creation of digital scarcity is accidental and its maintenance is due to a failure in governance. A prominent example is the dearth of IPv4 (Internet Protocol version 4) addresses (Rodriguez, 2012). The loom- 
ing shortage has been apparent since the 1990s, and yet the coordinated migration to the newer IPv6 has yet to be achieved on a large scale.

Bandwidth meanwhile is a hotly contested commodity and the ability to impose artificial scarcity on specific customers or types of content is subject to intense legal wrangling (Smith, 2010). Indeed, the imposition of limitations on bandwidth has been used as a means to clamp down on peer-to-peer file sharing or to degrade performance of a competitor's services, known as throttling. In response, the notion of 'net neutrality' emerged as part of a campaign aiming to protect the free flow of data and ensure that internet providers are not legally allowed to limit internet access based on content or source or usage (Wu, 2003; for an overview, see Finley, 2020).

\section{Digital scarcity in the age of blockchains}

In the context of the Bitcoin blockchain, digital scarcity refers to the limitation on the total supply of bitcoin. In contrast to the previous meaning, access to data is not restricted, and indeed the network relies on the blockchain data being freely available for anyone to copy in order to function securely. What determines a specific bitcoin is thus not its uniqueness as a piece of data, but rather its function as a verified entry in a distributed ledger.

It should also be noted that while both the cryptocurrency ether (on the Ethereum blockchain) and bitcoin (on the Bitcoin blockchain) are finite. The total amount of ether rises linearly whereas the total amount of bitcoin rises at a linear rate that is then halved every four years, so that the total number of bitcoins asymptotically approaches 21 million. As both blockchains enable finite transfers of a finite amount of digital currency, and the total amount in question grows only (sub-)linearly, they both exhibit digital scarcity and are not subject to uncontrolled inflation brought about by an out-of-control increase in money supply.

There is a further aspect of scarcity inherent in Bitcoin (and indeed any blockchain). Just as the bitcoin supply is limited to 21 million, so too is the Bitcoin network limited to seven transactions per second. These are both examples of digital scarcity, but while the former limit is entirely arbitrary and determined in protocol designs, the latter cannot be arbitrarily raised as it is bounded by bandwidth and processing constraints.

In the digital realm, data can be copied, databases re-indexed and values of variables can be changed-at least in principle. As the copyright battles of the 1990s 
and early 2000s made clear, maintaining digital data scarcity by preventing copies is nearly impossible. Copying data is just too easy and ubiquitous. However, establishing referential scarcity, where references are ledger/database entries (and the referents are anything from cryptocurrencies to cryptokitties), is possible as long as it can be credibly established that the scarcity will be maintained and the rules adhered to. The crucial aspect of referential scarcity is not control over data availability, but control over manipulation of the data in question. This was the innovation of Bitcoin and the invention of the blockchain as a decentralised ledger technology.

With the invention of Bitcoin, digital scarcity could be established without the need for a central entity to enforce it. Instead, the network uses cryptographic hash cycles (mining) in order to agree on, maintain and enforce a record of valid transaction data. Cryptocurrencies are not the first databases with finite number entries, but they are the first in which changes to the entries cannot be forced by the entities providing the computing infrastructure. The notion that centralised control over a database is necessary to ensure digital scarcity was thus overturned. 1

As more advanced and general-purpose blockchain networks such as Ethereum appeared, the scope for scarce ledger entries grew. Aside from scarcity of cryptocurrencies and currency-like 'tokens', a new class of 'unique digital items' known as non fungible tokens, or NFTs have appeared. These range from formal claims of ownership over a real-world (offline) asset, to purely digital collectibles (see, for example, Serada, Sihvonen, and Harviainen, 2020).

The rise of NFTs has led to experiments with new types of digital property where 'the broader intention does not appear to be to reduce the circulation and reproduction of the work, but instead to create titles and derivatives from its use and circulation' (O'Dwyer, 2020, p. 876). This for example implies producing a digital 'original' where its source and provenance is considered important enough to be able to acquire value as a 'unique' digital object, but where 'copies' can nevertheless circulate freely. However, there are considerable doubts about whether the possibilities afforded by distributed ledgers for new forms of digital scarcity will challenge much of the economic dynamics of property rights, or financial speculation and benefit producers of digital goods (Zeilinger, 2018; Lotti, 2016).

1. Meanwhile, in some cases centralised control does not guarantee the maintenance of digital scarcity either. This is evident not least from the Diablo 3 game: although the publisher-Blizzard Entertainment-nominally had complete control over all aspects of the game, they could not forestall runaway hyperinflation in the in-game economy (Mises, 2013). 


\section{Issues currently associated with the term}

At the time of writing, the culture around blockchains is still young, and it remains highly politicised and polarised. This polarisation contributes to the confusion surrounding digital scarcity as it relates to ideas of value. Proponents of Bitcoin in particular argue that it is the limited supply of bitcoins (and that alone) that gives them 'intrinsic' value whereas supporters of other blockchains (such as Ethereum, Cardano, Polkadot) argue that utility of the network, its 'extrinsic' value, is far more important. From their perspective, the limited performance of blockchain networks, which to the Bitcoin network is a feature, in fact inhibits the usability of the network and therefore growth of value.

In the context of the Bitcoin blockchain, digital scarcity tends to refer to the limitation on the total supply of bitcoin. The single-minded focus on Bitcoin's supply is not without precedent. New bitcoins are created in a staggered process, intended to replicate the dynamics of gold-it is increasingly hard to find, and the total supply is limited. Media theorist Golumbia (2016) traces these ideas in Bitcoin via the Austrian school of economics to right-of-centre US monetary ideas (and hard right conspiracy theories) that view the governance of money supply with deep suspicion. This line of thought views the government's very purpose as being the theft of ordinary people's wealth by printing money and causing hyperinflation. The broader consensus however is that good monetary governance, rather than no governance, is key to addressing not only hyperinflation but also other economic concerns. The correlation of hyperinflation with money supply draws on the Quantity Theory of Money (QTM). There are several real world examples of hyperinflation, from Zimbabwe (Ncube, 2019) to video games (Earle, 2013; Knowles et al., 2015, p. 248). But where proponents draw on QTM as a reason for absolute monetary and digital currency scarcity, critics - most notably of the school of Modern Monetary Theory, argue that money supply is not the main issue of concern, but rather how the supply is governed and what it is directed towards (Kelton, 2020).

\section{Conclusion}

Digital scarcity describes a credibly maintained limitation, imposed through software, of digital information, goods or services that may be accessed and used entirely digitally. This includes limitations to entries in a ledger or database (including cryptocurrency entries in a blockchain or top-level domains in the Domain Name System), as well as limitations in access to computing resources such as network addresses, bandwidth, or (again in the context of blockchains) transactionsper-second, wherever these limits go beyond the physical limits imposed by hard- 
ware. The motivations for engineering digital scarcity tend to be in order to support business models that profit from scarcity or uniqueness in the digital realm.

Older usage of the term includes physical limitations in processing power and bandwidth, and limitations in physical access to computing devices and computing services. Since in such cases, scarcity is not imposed through software, it is included in the history of the term but not in the current definition.

\section{References}

Andersson, J. (2011). The origins and impacts of the Swedish file-sharing movement: A case study. Critical Studies in Peer Production (CSPP), 1(1), 1-18. http://urn.kb.se/resolve?urn=urn:nbn:se:sh:div a-22494

Chaudhry, V., \& Shipp, T. (2005). Rethinking the digital divide in relation to visual disability in india and the united states: Towards a paradigm of 'Information Inequity'. Disability Studies Quarterly, 25(2). https://doi.org/10.18061/dsq.v25i2.553

Earle, P. C. (2013). A Virtual Weimar: Hyperinflation in a Video Game World [Blog post]. Mises Institute, Mises Daily Articles. https://mises.org/library/virtual-weimar-hyperinflation-video-game-wo rld

Finley, K. (2020, May). The WIRED guide to net neutrality. WIRED. https://www.wired.com/story/guid e-net-neutrality/

Fortin, M. (2007). Digital Scarcity: Does It Still Convert? Personal Blog [Blog post]. Michel Fortin's Blog. https://web.archive.org/web/20071217220308/https://michelfortin.com/digital-scarcity-doesit-still-convert/

Golumbia, D. (2016). The politics of Bitcoin. Software as right-wing extremism. University of Minnesota Press.

Hammersley, B. (2003, July 10). Radio active revolution. The Guardian. https://www.theguardian.co m/technology/2003/jul/10/onlinesupplement2

Kelton, S. (2020). The deficit myth, modern monetary theory and the birth of the people's economy. John Murray.

Knowles, I., Castronova, E., \& Ross, T. (2015). Video games, virtual worlds and economics. In R. G. Picard \& S. S. Wildman (Eds.), Handbook on the Economics of the Media. https://doi.org/10.4337/978 0857938893.00018

Liew, J. (2008, January 28). Three use cases for virtual goods [Blog post]. Lightspeed Venture Capital Partners. https://lsvp.wordpress.com/2008/01/28/three-use-cases-for-virtual-goods/

Lotti, L. (2016). Contemporary art, capitalization and the blockchain: On the autonomy and automation of art's value. Finance and Society, 2(2), 96. https://doi.org/10.2218/finsoc.v2i2.1724

Masnik, M. (2006a). Economics of abundance getting some well deserved attention. In Techdirt. http s://www.techdirt.com/articles/20061026/102329.shtml 
Masnik, M. (2006b). In a world where everything is digital, economics gets screwy fast. In Techdirt. h ttps://www.techdirt.com/articles/20061114/181724.shtml

Ncube, M. (2019, August 28). Introducing a new currency was Zimbabwe's only viable option. Financial Times. https://www.ft.com/content/f3e298c2-c8e7-11e9-a1f4-3669401ba76f

O'Dwyer, R. (2019). Limited edition: Producing artificial scarcity for digital art on the blockchain and its implications for the cultural industries. November 20, 2018. Convergence: The International Journal of Research into New Media Technologies, 26(4), 874-894. https://doi.org/10.1177/13548565 18795097

Oram, A. (Ed.). (2001). Peer-to-peer: Harnessing the benefits of a disruptive technology (1st ed.). O'Reilly.

Perzanowski, A., \& Schultz, J. (2016). The end of ownership: Personal property in the digital economy. MIT Press. https://doi.org/10.7551/mitpress/9780262035019.001.0001

Resnikoff, P. (2007, August 8). Bronfman ponders digital scarcity, retreads strategy. Digital Music News. https://www.digitalmusicnews.com/2007/08/08/warner-2/

Rodriguez, R. (2012, November). IPv4 scarcity [Blog post]. Internet Society. https://www.internetsoci ety.org/blog/2012/11/ipv4-scarcity/

Roemer, R., Schear, N., Dahlstrom, D., \& Farrington, N. (2006). Piracy in the digital age. University of Washington, Computer Science \& Engineering. https://courses.cs.washington.edu/courses/csep590 a/06au/projects/digital-piracy.pdf

Serada, A., Sihvonen, T., \& Harviainen, J. T. (2020). CryptoKitties and the new ludic economy: How blockchain introduces value, ownership, and scarcity in digital gaming. Games and Culture. https://d oi.org/10.1177/1555412019898305

See also conference proceedings: http://www.digra.org/digital-library/publications/cryptokitties-an d-the-new-ludic-economy-how-blockchain-introduces-value-ownership-and-scarcity-in-the-digitalworld/

Smith, C. E. (2010). Net neutrality, full throttle: Regulation of broadband internet service following the Comcast/Bittorrent dispute. Santa Clara Law Review, 50(2), 569-605. https://digitalcommons.la w.scu.edu/lawreview/vol50/iss2/7/

Swartz, A., \& Lessig, L. (2016). The boy who could change the world: The writings of Aaron Swartz. Verso.

Weinberger, D. (2003, March 13). The myth of interference. Salon. https://www.salon.com/2003/03/1 2/spectrum/

Wikipedia contributors. (2021a). Sex.com. In Wikipedia. Wikimedia Foundation. https://en.wikipedi a.org/w/index.php?title=Sex.com\&oldid=1013006654\#History

Wikipedia contributors. (2021b). Spectrum auction. In Wikipedia. Wikimedia Foundation. https://e n.wikipedia.org/w/index.php?title=Spectrum_auction \&oldid=1015746929

Wu, T. (2003). Network neutrality, broadband discrimination columbia law school scholarship archive. Journal of Telecommunications and High Technology Law, 2, 141-179. https://scholarship.la w.columbia.edu/faculty_scholarship/1281/

Zeilinger, M. (2018). Digital art as "Monetised graphics": Enforcing intellectual property on the 
blockchain. Philosophy \& Technology, 31(1), 15-41. https://doi.org/10.1007/s13347-016-0243-1

Published by

IIIIII

I O Alexander von Humbold

INSTITUTE FOR INTERNET

AND SOCIETY in cooperation with

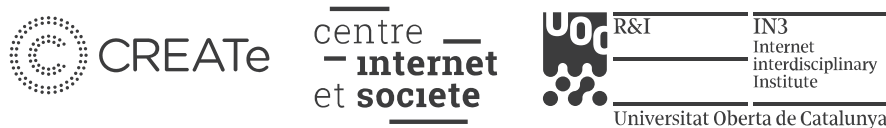

